\title{
Clinical Investigation of Patients with Brain Damage and Incidental Occurrence of Simultanagnosia
}

\section{Yasutaka Kobayashi MD ${ }^{1^{*}}$ and Shunsuke Tomizawa $\mathrm{AS}^{2}$}

${ }^{1}$ Department of Rehabilitation Medicine, Fukui General Hospital, Fukui, Japan

${ }^{2}$ Department of Rehabilitation Medicine, Fukui General Clinic, Fukui, Japan

\begin{abstract}
Purpose: The aim of this study was to investigate the clinical characteristics of patients with brain damage and incidental occurrence of simultanagnosia.

Methods: Among patients with brain damage who visited Fukui General Clinic for undergoing rehabilitation between 2012 and 2017, 16 patients with incidental occurrence of simultanagnosia were investigated. With reference to Ohigashi's classifications, brain damage was classified into the following forms: attentive form, perceptual form, or semantic form; clinical characteristics, symptoms in daily life, and imaging characteristics in patients with each form of brain damage were investigated. Results: There were nine patients in the attentive form group, four in the perceptual form group, and three in the semantic form group. All patients in the attentive form group had right parietal lobe lesions, with six exhibiting unilateral spatial neglect and three exhibiting optic ataxia. All four patients in the perceptual form group had left temporo-occipital lobe lesions and exhibited slow processing speeds that affected their daily lives. All three patients in the semantic form group were elderly and had brain atrophy.

Conclusion: We investigated lesion distribution and complicating symptoms in patients with brain damage and incidental occurrence of simultanagnosia based on the form of brain damage. In the attentive form group, unilateral lesions in the right parietal lobe had developed. Detailed interviews regarding daily life activities and detailed evaluation performed with the likelihood of simultanagnosia kept in mind are necessary when examining patients with brain damage.
\end{abstract}

Keywords: Simultanagnosia; Semantic form; Perceptual form; Attentive form; Clinical characteristics; Magnetic resonance imaging

\section{Introduction}

Multiple forms of simultanagnosia exist, such as the form in which the overall meaning of a situational drawing is not understood despite the visual recognition of details [1] and that in which it is difficult to simultaneously recognize multiple visual targets $[2,3]$. Some reports have indicated that lesions responsible for simultanagnosia are bilateral in the parietal, occipital, and temporal lobes [4-6]. As described above, there are multiple concepts for simultanagnosia, and prejudice that simultanagnosia occurs by bilateral brain lesions seems to be one of the reasons for the small number of reports on simultanagnosia. Moreover, it is difficult to diagnose simultanagnosia further because brain damage has many neurological symptoms. While simultanagnosia is a relatively rare condition, symptoms suggestive of the condition are commonly encountered in clinical settings.

In the present study, we summarized the clinical characteristics of suspected cases of simultanagnosia in patients who underwent rehabilitation.

\section{Subjects and Methods}

Among patients with brain damage who visited Fukui General Clinic to undergo rehabilitation between 2012 and 2017, 16 patients with incidental occurrence of simultanagnosia (10 men and 6 women, mean age: $58.6 \pm 16.2$ years) were included in the present study. Inclusion criteria were as follows: (1) history of brain damage and (2) confirmed simultanagnosia from episodes in daily life or rehabilitation and neuropsychological tests. Individuals with moderate-to-severe dementia were excluded. The patients were interviewed in detail regarding situations suggestive of simultanagnosia in their daily lives; their higher brain function was evaluated using neuropsychological testing, and complicating symptoms were confirmed. For neuropsychological testing, intelligence was evaluated using Wechsler Adult Intelligence Scale-III (WAIS-III) [7], memory was evaluated using the Rivermead Behavioural Memory Test (RBMT) [8], and attention was evaluated using the Trail Making Test (Part A) (TMT-A) [9]. The patients were divided into three groups according to Ohigashi's [10] classification based on explanations of situational images, symptoms in daily life, and findings during rehabilitation. Brain lesions were confirmed by performing head computed tomography or head magnetic resonance imaging (MRI) in all patients. This study was screened and approved by the Institutional Review Board of Nittazuka Health and Welfare Center (29-109). None of the authors have conflicts of interest in relation to this study.

\section{Results}

\section{Disease form and symptoms in daily life}

The disease form was semantic in three patients, perceptual in four patients, and attentive in nine patients. Although each patient classified

*Corresponding author: Yasutaka Kobayashi, Department of Rehabilitation Medicine Fukui General Hospital 58-16-1, Egami-cho, Fukui City, Fukui 910-8561, Japan, Tel: +81-776-59-1300; Fax: +81-776-59-2538; Email: yasutaka_k@mri.biglobe.ne.jp

Received February 15, 2018; Accepted March 02, 2018; Published March 08, 2018

Citation: Kobayashi Y, Tomizawa S (2018) Clinical Investigation of Patients with Brain Damage and Incidental Occurrence of Simultanagnosia. Brain Disord Ther 7 244. doi: 10.4172/2168-975X.1000244

Copyright: (c) 2018 Kobayashi Y, et al. This is an open-access article distributed under the terms of the Creative Commons Attribution License, which permits unrestricted use, distribution, and reproduction in any medium, provided the original author and source are credited. 
as having the semantic form was thought to have simple cognitive decline in their daily lives, they were found to have simultanagnosia because they were able to understand parts of the situation but were unable to comprehend the overall situation while performing cognitive tasks. While slow visual recognition speeds affected the daily lives of those in the perceptual form group, these patients were ultimately able to recognize the target. For the patients in attentive form group, their inability to simultaneously focus their attention on multiple targets affected their daily lives and task execution (Tables 1 and 2).

\section{Age}

The mean age of the patients was $80.0 \pm 7.5$ years in the semantic form group, $51.0 \pm 17.8$ years in the perceptual form group, and $54.9 \pm$ 11.9 years in the attentive form group, indicating that the patients in the semantic form group were more elderly than those in the other groups (Table 1).

\section{Complicating symptoms}

Constructional disturbance was noted in six of the nine patients in the attentive form group, two of the four patients in the perceptual form group, and one of the three patients in the semantic form group. Of the patients in the attentive form group, left unilateral spatial neglect was noted in six patients and optic ataxia was noted in three. Aphasia was noted in two patients in the perceptual form group and in one patient semantic form group. Visual field defects were also noted in two patients in the attentive form group and in one patient in the semantic form group. Among these three patients, one patient in the attentive form group and one in the semantic form group also had scene agnosia (Table 1).

\section{Neuropsychological test findings}

In six of the nine patients in the attentive form group, Performance IQ (PIQ), Perceptual Organization (PO), and Processing Speed (PS) scores in WAIS-III decreased. Moreover, RBMT scores decreased in three of these patients, and TMT-A duration was prolonged in one patient. WAIS-III scores in the perceptual form group decreased in the three patients who were able to undergo testing, with particularly decreased scores noted for PS. RBMT scores were decreased in all four patients in the perceptual form group, but TMT-A duration was found to be maintained in the two patients who were able to undergo this test. PIQ, PO, and RBMT scores were also found to be decreased in the one patient in the semantic form group who was able to undergo testing (Table 1).

\section{Underlying diseases}

Although the underlying disease was cerebrovascular disease in many patients, one patient in the semantic form group had Alzheimer's disease, one each in the perceptual form group had anoxic brain injury and a brain tumor, and one in the attentive form group had anoxic brain injury (Table 3).

\section{Lesion sites}

Among patients in the semantic form group, one exhibited left parietal lobe cerebral infarction and left temporo-occipital lobe atrophy, one exhibited right temporo-occipital lobe cerebral infarction and bilateral parietal lobe atrophy, and one patient with Alzheimer's disease exhibited overall brain atrophy. Among those in the perceptual form group, one exhibited left parietal lobe and left temporo-occipital lobe cerebral hemorrhage, one exhibited bilateral parietal lobe and bilateral temporo-occipital lobe cerebral infarction, one exhibited a right parietal lobe brain tumor and bilateral temporo- occipital lobe atrophy, and one with anoxic brain injury exhibited overall brain atrophy. Among those in the attentive form group, six exhibited right parietal lobe cerebral infarction or cerebral hemorrhage, two exhibited right parietal and temporo-occipital lobe cerebral hemorrhage, and one

\begin{tabular}{|c|c|c|c|c|c|c|c|c|c|c|c|c|c|}
\hline & \multirow{2}{*}{$\begin{array}{l}\text { Age } \\
(y)\end{array}$} & \multirow{2}{*}{ Sex } & \multirow{2}{*}{$\begin{array}{c}\text { Clinical form of } \\
\text { simultanagnosia }\end{array}$} & \multirow{2}{*}{ Complicated symptoms } & \multicolumn{7}{|c|}{ WAIS-III } & \multirow{2}{*}{$\begin{array}{l}\text { RBMT- } \\
\text { SPS }\end{array}$} & \multirow{2}{*}{$\begin{array}{l}\text { TMT-A } \\
\text { (s) }\end{array}$} \\
\hline & & & & & VIQ & $\mathrm{PIQ}$ & $\mathrm{FIQ}$ & VC & $\mathrm{PO}$ & WM & PS & & \\
\hline Case 1 & 62 & M & Attentive form & $\begin{array}{l}\text { unilateral spatial neglect, constructional disturbance, } \\
\text { It. hemiparesis }\end{array}$ & 95 & 52 & 71 & 100 & 57 & 81 & 52 & 0 & 158.5 \\
\hline Case 2 & 71 & $M$ & Attentive form & optic ataxia & 104 & - & - & 112 & - & 85 & 88 & 7 & - \\
\hline Case 3 & 45 & $\mathrm{~F}$ & Attentive form & $\begin{array}{c}\text { unilateral spatial neglect, constructional disturbance, } \\
\text { optic ataxia, It. hemiparesis }\end{array}$ & 76 & 50 & 61 & 92 & 55 & 56 & 52 & 1 & 210.9 \\
\hline Case 4 & 48 & M & Attentive form & $\begin{array}{c}\text { unilateral spatial neglect, constructional disturbance, } \\
\text { visual field defects, It. hemiparesis }\end{array}$ & 91 & 59 & 72 & 95 & 61 & 62 & 60 & 10 & 65.9 \\
\hline Case 5 & 45 & M & Attentive form & $\begin{array}{l}\text { unilateral spatial neglect, } \\
\text { It. hemiparesis }\end{array}$ & 101 & 80 & 91 & 102 & 83 & 102 & 63 & 8 & 53.4 \\
\hline Case 6 & 73 & $M$ & Semantic form & visual field defects, scene agnosia & 100 & 80 & 91 & 90 & 79 & 111 & 92 & 7 & 77.8 \\
\hline Case 7 & 36 & $\mathrm{~F}$ & Attentive form & amnesia, executive dysfunction & 90 & 84 & 86 & 93 & 79 & 88 & 84 & 0 & 35.5 \\
\hline Case 8 & 79 & $\mathrm{~F}$ & Semantic form & amnesia & - & - & - & - & - & - & - & - & 95.1 \\
\hline Case 9 & 63 & M & Perceptual form & - & 81 & 64 & 70 & 80 & 66 & 79 & 60 & 6 & 64.7 \\
\hline Case 10 & 28 & M & Perceptual form & constructional disturbance, aphasia & - & 48 & - & - & 52 & - & 50 & 1 & - \\
\hline Case 11 & 88 & $\mathrm{~F}$ & Semantic form & $\begin{array}{l}\text { constructional disturbance, aphasia, } \\
\text { rt. hemiparesis }\end{array}$ & - & - & - & - & - & - & - & - & - \\
\hline Case 12 & 68 & $\mathrm{~F}$ & Attentive form & $\begin{array}{c}\text { unilateral spatial neglect, constructional disturbance, } \\
\text { visual field defects, scene agnosia }\end{array}$ & 94 & 67 & 79 & 95 & 79 & 85 & 52 & 8 & 180.2 \\
\hline Case 13 & 58 & M & Attentive form & unilateral spatial neglect, constructional disturbance & 107 & 99 & 104 & 109 & 101 & 100 & 97 & 9 & 39.5 \\
\hline Case 14 & 46 & M & Perceptual form & - & 84 & 62 & 70 & 92 & 63 & 72 & 52 & 4 & 91.8 \\
\hline Case 15 & 61 & M & Attentive form & constructional disturbance, optic ataxia & 100 & 97 & 98 & 104 & 99 & 98 & 86 & 11 & 28.8 \\
\hline Case 16 & 67 & M & Perceptual form & constructional disturbance, aphasia, rt. hemiparesis & - & - & - & - & - & - & - & 7 & - \\
\hline
\end{tabular}

Table 1: Age, sex, disease form, complicating symptoms, and neuropsychological testing. 
Citation: Kobayashi Y, Tomizawa S (2018) Clinical Investigation of Patients with Brain Damage and Incidental Occurrence of Simultanagnosia. Brain Disord Ther 7: 244. doi: 10.4172/2168-975X.1000244

Symptoms in daily life

Case 1 He cannot follow the sequence of numbers representing items available from the vending machine and skips some numbers on the machine.

Case 2 When he tries to read a passage, he partitions a sentence in a wrong way and fails to identify a sentence.

Case 2 When he tries to recognize a figure, he only looks at its parts and fails to identify the whole.

Case 3 Reading a newspaper article, she skips to a wrong line.

She cannot read even when letter-by-letter reading is tried.

Case 4 When he tries to carry out a task, he just starts with what has caught his eyes.

Case 5 When he attends the housework, he just starts with what has caught his eyes.

Case 6 He cannot recognize the overall pattern of the blocks he has stacked.

Case 7 If she is given an instruction while driving a car in the middle of the road she suddenly runs the car into the side wall.

Case 8 She cannot recognize what is finally represented by the picture that she has colored.

Case 9 When he looks at an object placed before him, it takes considerable time to recognize the whole.

Case 10 He cannot find the faucet in the toilet.

Case 10 He cannot recognize what is pointed at and moves his eyes toward other points, so it takes considerable time to find it.

Case 11 She cannot recognize the overall pattern of the blocks she has stacked.

While reading a sentence of a book, she suddenly jumps to the next page.

Case 12 Reading a newspaper article, she skips to a wrong line.

She cannot read even when letter-by-letter reading is tried.

While reading a sentence of a book, he suddenly jumps to the next page.

Case 13 Reading a newspaper article, he skips to a wrong line.

He cannot read even when letter-by-letter reading is tried.

Case 14 When he attends the housework, he just starts with what has caught his eyes.

Case 15 As he fails to recognize the main dish and side dishes simultaneously served on the table, he cannot eat the meal smoothly.

Case 16 When he attends the housework, he just starts with what has caught his eyes.

Case 16 While walking, he hits his head against the wall without noticing it.

Table 2: Symptoms in daily life.

\begin{tabular}{|c|c|c|c|c|c|c|c|}
\hline & \multirow{3}{*}{ Disease } & \multicolumn{6}{|c|}{ Affected area } \\
\hline & & \multicolumn{4}{|c|}{ Parietal lobe } & \multicolumn{2}{|c|}{ Temporo-occipital lobe } \\
\hline & & Side & Superior parietal lobule & Intraparietal sulcus & Inferior parietal lobule & Side & Inferior surface \\
\hline Case 1 & $\mathrm{ICH}$ & $\mathrm{R}$ & + & + & + & $\mathrm{R}$ & + \\
\hline Case 2 & $\mathrm{Cl}$ & $\mathrm{R}$ & + & + & + & & - \\
\hline Case 3 & $\mathrm{Cl}$ & $\mathrm{R}$ & + & + & + & & - \\
\hline Case 4 & $\mathrm{ICH}$ & $\mathrm{R}$ & + & + & + & $\mathrm{R}$ & + \\
\hline Case 5 & $\mathrm{ICH}$ & $\mathrm{R}$ & + & + & + & & - \\
\hline Case 6 & $\mathrm{Cl}$ & $\mathrm{RL}$ & + (atrophy) & + (atrophy) & + (atrophy) & $\mathrm{R}$ & + \\
\hline Case 7 & $\mathrm{ABI}$ & $\mathrm{RL}$ & + (atrophy) & + (atrophy) & + (atrophy) & $\mathrm{RL}$ & + (atrophy) \\
\hline Case 8 & $A D$ & $\mathrm{RL}$ & + (atrophy) & + (atrophy) & + (atrophy) & $\mathrm{RL}$ & + (atrophy) \\
\hline Case 9 & BT & $\mathrm{R}$ & + & + & + & $\mathrm{RL}$ & + (atrophy) \\
\hline Case 10 & $\mathrm{ICH}$ & L & + & + & + & L & + \\
\hline Case 11 & $\mathrm{Cl}$ & L & + & + & + & L & + (atrophy) \\
\hline Case 12 & $\mathrm{ICH}$ & $\mathrm{R}$ & + & + & + & & - \\
\hline Case 13 & $\mathrm{ICH}$ & $\mathrm{R}$ & - & + & + & & - \\
\hline Case 14 & $\mathrm{ABI}$ & $\mathrm{RL}$ & + (atrophy) & + (atrophy) & + (atrophy) & $\mathrm{RL}$ & + (atrophy) \\
\hline Case 15 & $\mathrm{Cl}$ & $R$ & + & + & + & & - \\
\hline Case 16 & $\mathrm{Cl}$ & $\mathrm{RL}$ & + & + & + & $\mathrm{RL}$ & + \\
\hline
\end{tabular}

Cl: Cerebral Infarction; ICH: Intracerebral Hemorrhage; ABI: Anoxic Brain Injury; BT: Brain Tumor; AD: Alzheimer's Disease

Table 3: Underlying diseases and lesion sites.

with anoxic brain injury exhibited overall brain atrophy. Figures 1a-1c shows the representative cases for each form (Table 3).

\section{Discussion}

The term simultanagnosia was first used by Wolpert [1] to describe the ability to correctly perceive individual elements of a picture made up of multiple elements without understanding the overall meaning of that picture. The term has also been used to describe symptoms such as those reported by Balint [2] and Luria [3] where a patient is unable to see more than one object at the same time. Ohigashi [10] classified this confusing condition into three forms: semantic form, perceptual form, and attentive form. The semantic form refers to simultanagnosia in which the overall meaning of a situational picture is not synthesized and was the form described by Wolpert [1]. This form is not accompanied by alexia, and patients exhibit no symptoms such as difficulty recognizing multiple targets or slowed exploration. However, patients are capable of partial recognition necessary to understand the meaning of the situational picture and can competently reproduce shapes. The perceptual form refers to simultanagnosia in which the speed of visual processing of multiple targets is decreased. Along with alexia characterized by letter-by-letter reading, patients can look at a situational picture in detail at a slow speed but are not quite 

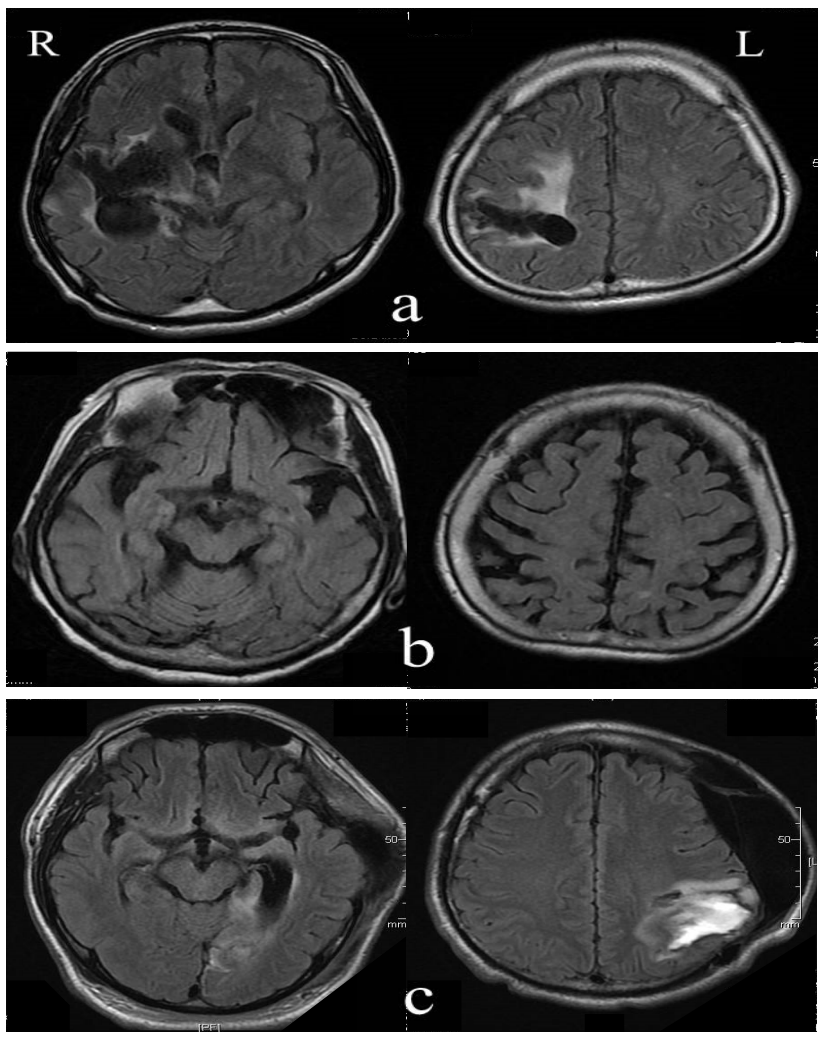

Figure 1: Head magnetic resonance imaging (FLAIR).

(a) Case 4 (attentive form): An old right putaminal hemorrhage is visible. The lesion extends from the basal ganglia to the right parietal and right temporo-occipital lobes.

(b) Case 8 (semantic form): Atrophy of the entire brain including the bilateral parietal and temporal lobes is visible.

(c) Case 10 (perceptual form): An old left subcortical hemorrhage is visible

The lesion extends to the left parietal and left temporo-occipital lobes.

able to comprehend the overall meaning. The case reported by Mondez et al. [11] is consistent with this form. The attentive form refers to simultanagnosia that arises secondary to a visual attentive disorder. The external world can only be fragmentally viewed, with objects suddenly disappearing and appearing to a patient. This is a well-known symptom of Bálint's syndrome. Recently, Dalrymple et al. [12] described attentive simultanagnosia as the "reduced spatial window of attention."

The attentive form is the most common form. According to the theory that this form is secondary simultanagnosia caused by visual attentive disorder [10], it should be possible to detect this simultanagnosia during attentional testing. However, we were unable to detect attentive simultanagnosia during simple testing by means such as the TMT-A used in this study. If detailed interviews about the daily lives of patients could be conducted like they were in our study, it might be possible to detect the condition more frequently. Although it took time for patients to find visual targets directly in front of them and to perceive the entire image, recognition of the image was ultimately possible in all of the four patients with perceptual simultanagnosia. Decreased visual processing speed of multiple targets therefore appears to exist in this form. The three semantic form patients varied in age from 73 to 88 and were clearly more elderly than the patients with the other two forms of simultanagnosia. This form of simultanagnosia can also be assigned to the specific form of associative visual agnosia and could have arisen as a result of the addition of organic lesions to agerelated cognitive decline.

Studies of the lesions responsible for simultanagnosia have used voxel-based morphometry and tractography [5] and functional MRI [6]. The former study detected bilateral impairment of the medial occipito-parietal junction, cuneus, and inferior intra-parietal sulcus, in addition to impairment of the underlying white matter tract, while the latter study detected impairment of the bilateral lateral and medial inferior parietal cortex that correlated to simultanagnosia. Tanemura et al. [13] reported that the semantic form according to Ohigashi's classifications is caused by left-dominant lesions in the bilateral medial occipito-temporal lobe, and that the attentive form is caused by bilateral lesions in the parieto-occipital lobe. The attentive form patients were comparable with patients with dorsal simultanagnosia reported by Farah [14]. This form is thought to be associated with lesions arising in the bilateral parieto-occipital lobe. All nine of the attentive form patients in this study exhibited right parietal lobe lesions, with only one patient exhibiting bilateral lesions. As previously mentioned, this form is thought to be simultanagnosia that arises secondary to a visual attentive disorder. The symptoms of unilateral lesions may be milder than those of bilateral lesions. In cases of unilateral lesions in which symptoms such as unilateral spatial neglect and aphasia appear in the foreground, it is possible that the symptoms of latent simultanagnosia might be overlooked during medical examinations, and the condition might instead become more noticeable in the patient's daily life over time. The perceptual form appears to be equivalent to the ventral simultanagnosia reported by Farah [14] and is likely caused by lesions on the lower surfaces of the left occipito-temporal lobe. Each of our three perceptual form patients exhibited lesions on the lower surface of the left parietal lobe and the symptoms were also anatomically explainable. The semantic form is comparable with the form reported by Wolpert and is thought to be a special form of associative visual agnosia. Lesions are usually found on the bilateral or on the left medial surfaces of the occipito-temporal lobe. Of our three patients, one exhibited bilateral temporo-occipital lobe lesions, one exhibited a left lesion and one exhibited a right lesion. Naccache et al. [15] reported a case of possible semantic simultanagnosia caused by a right parietal lobe lesion. Further data of more patients needs to be gathered to determine the relationship between simultanagnosia form and lesion site.

In the large majority of cases, the underlying cause of simultanagnosia was localized lesions such as cerebrovascular disease or a brain tumor. However, the semantic form patients included one case of Alzheimer's disease, while the perceptual and attentive form patients each included one patient with anoxic brain injury. In these diseases where the entire brain is impaired, the form of simultanagnosia appears to differ depending on the extent and progression of the underlying disease. Even in cases of cerebrovascular disease and brain tumor, examples of concomitant atrophy of distant sites have been observed, which points to the need for detailed imaging when simultanagnosia is suspected.

Neuropsychological testing in this study revealed the decline in intelligence and memory impairment. The decline in PIQ was striking, especially in comparison with Verbal IQ, and this appeared to be the result of a large number of patients with lesions in the right cerebral hemisphere. The decrease in PS among perceptual form patients may reflect the decline that occurred in the visual processing speed of multiple targets. Furthermore, contrary to expectations, the TMT-A duration was prolonged in just one patient in the attentive form group. Our results suggest that evaluation of attentional function in simultanagnosia might be impossible using simple tests such as the TMT-A and that more detailed attentional function testing is needed. 
Many of our patients had neuropsychological complications. Patients with aphasia, unilateral spatial neglect, constructional disturbance, and visual field defects, which are conditions often encountered in clinical settings, may include a considerable number of individuals with simultanagnosia. The underlying diseases in this study also included Alzheimer's disease and anoxic brain injury. There are reports on visual attentive disorder accompanying Alzheimer's disease [16], and various cognitive dysfunctions including attention disorder have been reported in Parkinson's disease [17]. Therefore, when extensive damage has occurred to the cerebral cortex, as in the case of neurodegenerative disorders, various neuropsychological symptoms may appear in the foreground and the lack of insight into the disease may impede the detection of simultanagnosia. Detailed interviews regarding daily life and detailed evaluation with the possibility of simultanagnosia in mind are therefore necessary when examining patients with brain damage. The limitations of this study include the small sample size and possible selection bias.

\section{Conclusion}

Simultanagnosia, including forms that cannot be detected in routine examinations, is relatively commonly encountered. The most common form of simultanagnosia is the attentive form and may present in cases of extensive brain damage as well as cases of local lesions. In addition to bilateral lesions, which are traditionally considered to be involved in simultanagnosia, patients may also present with unilateral lesions. Detailed interviews regarding daily life activities and detailed evaluation done with the possibility of simultanagnosia kept in mind are necessary when examining patients with brain damage.

\section{References}

1. Wolpert I (1924) Die Simultanagnosie - Störung der Gesamtausffassung. Z. gesamte Neurol Psychiat 93: 397-415.

2. Bálint R (1909) Seelenlähmung des Schauens, optische Ataxie, räumliche Störung der Aufmerksamkeit. Mschr Psychiat Neurol 25: 51-81.
3. Luria AR (1959) Disorders of 'simultaneous perception' in a case of bilateral occipitoparietal brain injury. Brain 82: 437-449.

4. Michel F, Henaff MA (2004) Seeing without the occipito-parietal cortex: Simultagnosia as a shrinkage of the attentional visual field. Behav Neurol 15 3-13.

5. Chechlacz M, Rotshtein P, Hansen PC, Riddoch JM, Deb S, et al. (2012) The neural underpinings of simultanagnosia: disconnecting the visuospatial attention network. J Cogn Neurosci 24: 718-735.

6. Himmelbach M, Erb M, Klockgether T, Moskau S, Karnath HO (2009) fMRI of global visual perception in simultanagnosia. Neuropsychologia 47: 1173-1177.

7. Fujita K, Maekawa H, Dairoku K, Yamanaka K (2007) The Japanese version of the Wechsler Adult Intelligence Scale-III. Nihon Bunka Kagakusha, Tokyo, Japan.

8. Watamori T, Hara H, Miyamori T, Eto $F(2002)$ The Japanese version of the Rivermead Behavioural Memory Test. Chiba Test Center, Tokyo, Japan.

9. Partington JE, Leiter RG (1949) Partington's pathway test. Psychological Service Center Journal 1: 11-20.

10. Ohigashi Y (2002) Some historical and methodological remarks in neuropsychology. Higher Brain Func Res 22: 215-220.

11. Mendez MF, Cherrier MM (1998) The evolution of alexia and simultanagnosia in posterior cortical atrophy. Cogn Behavioral Neurol 11: 76-82.

12. Dalrymple KA, Barton JJS, Kingstone A(2013)A world unglued: simultanagnosia as a spatial restriction of attention. Front Hum Neurosci 7: 145.

13. Tanemura J, Tsubahara A (2014) Visual perception-simultanagnosia. Clin Neurosci 32: 157-160.

14. Farah MJ (1990) Visual Agnosia. MIT Press, Cambridge, Massachusetts London.

15. Naccache L, Slachevsky A, Levy R, Dubois B (2000) Simultanagnosia in a patient with right brain lesions. J Neurol 247: 650-651.

16. Coslet HB, Stark M, Rajaram S, Saffran EM (1995) Narrowing the spotlight: a visual attentional disorder in presumed Alzheimer's disease. Neurocase 1 305-318.

17. Amarendranath $C$, Dhilleswara $R$, Jagadeesh VR, Tuhin SB, Arun KR, et al. (2017) Cognitive and psychological anomalies in Parkinson's disease: An insight into non-motor characteristic features. Neuropsychiatry 7: 1069-1080. 